\title{
Production-inventory games and PMAS-games: Characterizations of the Owen point
}

\author{
Luis A. Guardiola ${ }^{\mathrm{a}, *}$, Ana Meca ${ }^{\mathrm{a}}$, Justo Puerto ${ }^{\mathrm{b}}$ \\ a Operations Research Center, Universidad Miguel Hernández, Edificio Torretamarit. Avda. de la Universidad s.n., \\ 03202 Elche (Alicante), Spain \\ ${ }^{\mathrm{b}}$ Facultad de Matemáticas, Universidad de Sevilla, 41012 Sevilla, Spain
}

Received 7 November 2006; received in revised form 28 August 2007; accepted 6 December 2007

\begin{abstract}
Production-inventory games were introduced in [Guardiola, L.A., Meca, A., Puerto, J. (2008). Production-Inventory games: A new class of totally balanced combinatorial optimization games. Games Econom. Behav. doi:10.1016/j.geb.2007.02.003] as a new class of totally balanced combinatorial optimization games. From among all core-allocations, the Owen point was proposed as a specifically appealing solution. In this paper we study some relationships of the class of production-inventory games and other classes of new and known games. In addition, we propose three axiomatic characterizations of the Owen point. We use eight axioms for these characterizations, among those, inessentiality and additivity of players' demands are used for the first time in this paper.
\end{abstract}

(C) 2008 Elsevier B.V. All rights reserved.

JEL classification: $\mathrm{C} 71$

MSC: 91A12; 90B05

Keywords: Production-inventory games; Owen point; Totally balanced combinatorial optimization games; Coreallocations

\footnotetext{
The research of the authors was partially supported by the Spanish Ministry of Education and Science, Junta de Andalucía and Generalitat Valenciana grant numbers: MTM2004-0909, HA2006-0121, MTM2005-09184-C02-02, MTM2007-67433-C02-01, ACOMP06/040, CSD2006-00032 and P06-FQM-01366. The authors acknowledge the useful comments made by the Associate Editor and the referee.

* Corresponding author.

E-mail addresses: luis.guardiola@alu.umh.es (L.A. Guardiola), ana.meca@umh.es (A. Meca), puerto@us.es (J. Puerto).
} 


\section{Introduction}

In this paper we focus on the class of production-inventory cooperative games, introduced in Guardiola et al. (2008). We consider a group of firms that produce indivisible goods over a finite planning horizon to cover a known demand. We assume that there exist three different types of costs: production, inventory holding and backlogging. The goal of each individual firm is to satisfy its entire demand over the planning horizon at a minimum operation cost. (This model is known in the O.R. literature as the Wagner-Whitin model, see Wagner and Whitin (1958).

Cooperation enters the model throughout coordination among firms. Specifically, if a group of firms forms a coalition (joint venture) then they will use the best technology among the members. This means that the members of that coalition produce, hold inventory and pay backlogged demand at the minimum cost of the coalition members. It is clear that the above coordination process induces savings and therefore, studying the problem of how to allocate the overall saving among the firms is a meaningful problem. This allocation problem can be modeled by a transferable utility cooperative game. In this game the worth of each coalition of firms is obtained solving the combinatorial optimization problem that results from the Wagner-Whitin model with production, inventory holding and backlogging costs induced by the members of the coalition. Other classes of combinatorial optimization games can be found in Deng et al. $(1999,2000)$ and the references therein.

The analysis of coordination in inventory problems is not new. Scanning the literature, one can find centralization inventory models analyzed from this point of view. Eppen (1979), Hartman et al. (2000), Hartman and Dror (2003, 2005), Slikker et al. (2005), and Müller et al. (2002) have treated cooperation in a news-vendor problem. A similar consideration for the continuous review inventory model is studied in Gerchak and Gupta (1991), Robinson (1993), and Hartman and Dror (1996). Tijs et al. (2005) studies a situation where one agent owns an amount of storage space and the other agents have some goods, part of which can be stored generating benefits. A general framework for the study of continuous time decentralized distribution systems is analyzed in Anupindi et al. (2001). The problem of sharing the benefits produced by full cooperation between agents is tackled by introducing a related cooperative game. Minner (2006) analyzes horizontal cooperation between organizations that have the opportunity to jointly replenish material requirements. In Meca et al. $(2003,2004)$ and Meca (2007) a group of firms dealing with the ordering and holding of a certain commodity (every individual agent's problem being an EPQ and EOQ problem, respectively), either decide to cooperate and make their orders jointly or consider coordination with regard to ordering and holding costs. Finally, Meca et al. (2007) studies a new class of totally balanced games that arise from inventory with temporary discounts. The interested reader is referred to Borm et al. (2001) for a detailed presentation of inventory games, as well as other Operations Research games.

Production-inventory games (henceforth: PI-games) were studied in Guardiola et al. (2008) where it is shown that this class of games is strictly included in the class of totally balanced games. It was also proven that the Owen set (the set of allocations that are achievable through dual solutions, see Owen (1975) and Gellekom et al. (2000)) reduces to a singleton. This fact motivates the name Owen point rather than Owen set within this class of games. Finally, Guardiola et al. (2008) also proves that the Owen point always belongs to the core of the game and that can be reached through a population monotonic allocation scheme. Hence, every PI-game is a non-negative cost game allowing for population monotonic allocation schemes (henceforth: PMAS-game). 
In this paper we prove that the class of PI-games coincides with the class of PMAS-games, and we provide an interesting relationship between PI-games and concave games. In addition, we present three different axiomatic characterizations of the Owen point. To achieve the two first characterizations we have kept in mind the work by Gellekom et al. (2000) in which the Owen set of linear production games is characterized. The third one, which is based on a population monotonicity property, is very natural due to the fact that the class of PI-games coincides with the class of non-negative cost games with a population monotonic allocation scheme.

The paper is organized as follows. We start by introducing definitions and notation in Section 2. In Section 3 we introduce the class of PMAS-games. There, we also study relationships with PI-games and other classes of games. Section 4 provides three characterizations of the Owen point. The paper finishes with a section devoted to draw some conclusions and final remarks.

\section{Preliminaries}

A TU cost game is a pair $(N, c)$, where $N=\{1,2, \ldots, n\}$ is a finite set of players, $\mathcal{P}(N)$ is the set of non-empty coalitions of $N$ and $c: \mathcal{P}(N) \rightarrow \mathbb{R}$ the characteristic function satisfying $c(\varnothing)=0$. The subgame related to coalition $S, c_{S}$, is the restriction of the mapping $c$ to the subcoalitions of $S$. We denote by $|S|$ the cardinal of set $S$, for all $S \subseteq N$. A game $(N, c)$ is said to be 0-monotone if it is monotone after 0-normalization, i.e., for all $S, S^{\prime} \subset N$ with $S \subset S^{\prime}$ we have $c(S)-\sum_{i \in S} c(\{i\}) \geq c\left(S^{\prime}\right)-\sum_{i \in S^{\prime}} c(\{i\})$. A player $i \in N$ is a veto player in the game $c$ if and only if $c(S)=0$ for all $S \subseteq N \backslash\{i\}$. For convenience we call a game with at least one veto player a veto game. Finally, $(N, c)$ is called a simple game whenever $c(S) \in\{0,1\}$ for all $S \subseteq N$.

A cost-sharing vector will be $x \in \mathbb{R}^{N}$ and, for every coalition $S \subseteq N$ we shall write $x_{S}:=\sum_{i \in S} x_{i}$, the cost-sharing of coalition $\mathrm{S}$ (where $x_{\varnothing}=0$ ). The core of the game $(N, c)$ consists of those cost-sharing vectors which allocate the cost of the grand coalition in such a way that every other coalition pays at most its cost by the characteristic function: Core $(N, c)=\left\{x \in \mathbb{R}^{N} / x_{N}=c(N)\right.$ and $x_{S} \leq c(S)$ for all $\left.S \subset N\right\}$. In the following, costsharing vectors belonging to the core will be called core-allocations. A cost game $(N, c)$ has a non-empty core if and only if it is balanced (see Bondareva (1963) or Shapley (1967)). It is a totally balanced game (Shapley and Shubik, 1969) if the core of every subgame is non-empty.

A well-known class of balanced games is the class of concave games (Shapley, 1971). A TUgame $(N, c)$ is concave if and only if $c(S \cup\{i\})-c(S) \geq c(T \cup\{i\})-c(T)$ for all players $i \in N$ and all pairs of coalitions $S, T \subseteq N$ such that $S \subseteq T \subseteq N \backslash\{i\}$. A population monotonic allocation scheme (see Sprumont (1990)), or PMAS, for the game $(N, c)$ is a collection of vectors $y^{S} \in \mathbb{R}^{S}$ for all $S \subseteq N, S \neq \varnothing$ such that $y^{S}(S)=c(S)$ for all $S \subseteq N, S \neq \varnothing$, and $y_{i}^{S} \geq y_{i}^{T}$ for all $S \subseteq T \subseteq N$ and $i \in S$. Finally, for a generic mathematical programming problem $(P)$, val $(P)$ denotes the optimal value of problem $(P)$.

A production-inventory situation (henceforth: PI-situation) is the one in which several agents facing each one a production-inventory problem, decide to cooperate to reduce costs. The cooperation is driven by sharing technologies in production, inventory carrying and backlogged demand. Thus, if a group of agents agree on cooperation then at each period they will produce and pay inventory carrying and backlogged demand at the cheapest costs among the members of the coalition. Formally, let $U$ be an infinite set, the universe of players. A PI-situation is a 3-tuple $(N, D, \Re)$ where $N \subset U$ is a finite set of players $(|N|=n)$, being $D$ an integer matrix of demands and $\Re=(H|B| P)$ is a cost matrix, so that

$$
D=\left[d^{1}, \ldots, d^{n}\right]^{\prime}, \quad H=\left[h^{1}, \ldots, h^{n}\right]^{\prime}, \quad B=\left[b^{1}, \ldots, b^{n}\right]^{\prime}, \quad P=\left[p^{1}, \ldots, p^{n}\right]^{\prime} ;
$$


where:

- $T$ is the planning horizon.

- $d^{i}=\left[d_{1}^{i}, \ldots, d_{T}^{i}\right] \geq 0, d_{t}^{i}=$ demand of the player $i$ during period $t, t=1, \ldots, T$.

- $h^{i}=\left[h_{1}^{i}, \ldots, h_{T}^{i}\right] \geq 0, h_{t}^{i}=$ unit inventory carrying costs of the player $i$ in period $t, t=1, \ldots, T$.

- $b^{i}=\left[b_{1}^{i}, \ldots, b_{T}^{i}\right] \geq 0, b_{t}^{i}=$ unit backlogging carrying costs of the player $i$ in period $t, t=1, \ldots, T$.

- $p^{i}=\left[p_{1}^{i}, \ldots, p_{T}^{i}\right] \geq 0, p_{t}^{i}=$ unit production costs of the player $i$ in period $t, t=1, \ldots, T$. The decision variables of the model, which are required to be integer quantities, are:

- $q_{t}=$ production during period $t$.

- $I_{t}=$ inventory at hand at the end of period $t$.

- $E_{t}=$ backlogged demand at the end of period $t$.

Note that we can associate with each PI-situation $(N, D, \mathfrak{R})$ a cost TU-game $(N, c)$ with characteristic function $c$ defined as follows: $c(\varnothing)=0$ and for any $S \subseteq N, c(S)=\operatorname{val}(\operatorname{PI}(S))$, where $(\mathrm{PI}(S))$ is the following problem

$$
\begin{aligned}
(\mathrm{PI}(S)) \quad \min & \sum_{t=1}^{T}\left(p_{t}^{S} q_{t}+h_{t}^{S} I_{t}+b_{t}^{S} E_{t}\right) \\
& \text { s.t. } \quad I_{0}=I_{T}=E_{0}=E_{T}=0, \\
& I_{t}-E_{t}=T_{t-1}-E_{t-1}+q_{t}-d_{t}^{S}, \quad t=1, \ldots, T . \\
& q_{t}, I_{t}, E_{t}, \text { non-negative, integer, } t=1, \ldots, T ;
\end{aligned}
$$

with

$$
p_{t}^{S}=\min _{i \in S}\left\{p_{t}^{i}\right\}, \quad h_{t}^{S}=\min _{i \in S}\left\{h_{t}^{i}\right\}, \quad b_{t}^{S}=\min _{i \in S}\left\{b_{t}^{i}\right\}, \quad d_{t}^{S}=\sum_{i \in S} d_{t}^{i} .
$$

Every cost TU-game defined in this way is what we call a production-inventory game. Guardiola et al. (2008) observes that the linear relaxation, $(\operatorname{LPI}(S))$ of the problem $(\operatorname{PI}(S))$, has integer optimal solutions provided that the demands are integer. It follows from standard duality theory of linear programming that the dual problem for any coalition $S \subseteq N$ is the following problem,

$$
\begin{aligned}
(\operatorname{DLPI}(S)) \quad \max & \sum_{t=1}^{T} d_{t}^{S} y_{t} \\
\text { s.t. } \quad & y_{t} \leq p_{t}^{S}, \quad t=1, \ldots, T, \\
& y_{t+1}-y_{t} \leq h_{t}^{S}, \quad t=1, \ldots, T-1, \\
& -y_{t+1}+y_{t} \leq b_{t}^{S}, \quad t=1, \ldots, T-1 .
\end{aligned}
$$

In order to illustrate the structure of the model, we consider a special case with two periods. Let $S$ be a coalition of players in $N$. The problem $\operatorname{LPI}(S)$ and its dual can be written as:

$$
\min \quad p_{1}^{S} q_{1}+p_{2}^{S} q_{2}+h_{1}^{S} I_{1}+b_{1}^{S} E_{1} \quad \text { and } \quad \max \quad d_{1}^{S} y_{1}+d_{2}^{S} y_{2}
$$$$
\text { s.t. }\left[\begin{array}{cccc}
1 & 0 & -1 & 1 \\
0 & 1 & 1 & -1
\end{array}\right]\left[\begin{array}{c}
q_{1} \\
q_{2} \\
I_{1} \\
E_{1}
\end{array}\right]=\left[\begin{array}{l}
d_{1}^{S} \\
d_{2}^{S}
\end{array}\right] \quad \text { s.t. }\left[\begin{array}{cc}
1 & 0 \\
0 & 1 \\
-1 & 1 \\
1 & -1
\end{array}\right]\left[\begin{array}{l}
y_{1} \\
y_{2}
\end{array}\right] \leq\left[\begin{array}{c}
p_{1}^{S} \\
p_{2}^{S} \\
h_{1}^{S} \\
b_{1}^{S}
\end{array}\right] \text {. }
$$

$q_{1}, q_{2}, I_{1}, E_{1} \geq 0$. 
Guardiola et al. (2008) proves that the optimal solution of problem $(\operatorname{DLPI}(S))$ is $y_{t}^{*}(S)=$ $\min \left\{p_{t}^{S}, \min _{k<t}\left\{p_{k}^{S}+h_{k t}^{S}\right\}, \min _{k>t}\left\{p_{k}^{S}+b_{t k}^{S}\right\}\right\}$, for all $t=1, \ldots, T$, with

$$
\begin{aligned}
p_{k}^{S} & = \begin{cases}p_{1}^{S} & \text { if } k<1, \\
p_{T}^{S} & \text { if } k>T,\end{cases} \\
h_{k t}^{S} & =\sum_{r=k}^{t-1} h_{r}^{S}, \quad \text { for any } k<t, t=2, \ldots, T ; \quad h_{k 1}^{S}=0, \quad k<1 . \\
b_{t k}^{S} & =\sum_{r=t}^{k-1} b_{r}^{S}, \quad \text { for any } k>t, t=1, \ldots, T-1 ; \quad b_{T k}^{S}=0, \quad k>T .
\end{aligned}
$$

Moreover, these optimal solutions satisfy the following property: $y_{t}^{*}(S) \geq y_{t}^{*}(R)$ for all $S \subseteq R \subseteq N$ and all $t \in\{1, \ldots, T\}$. The characteristic function of PI-games can be rewritten as follows: for any $\varnothing \neq S \subseteq N, c(S)=\sum_{t=1}^{T} d_{t}^{S} y_{t}^{*}(S)$.

The reader may note that PI-games are not concave in general (see Example 4.4 in Guardiola et al. (2008)). In these games the allocation $\left(\sum_{t=1}^{T} d_{t}^{i} y_{t}^{*}(N)\right)_{i \in N}=D y^{*}(N)$ is called the Owen point, and it is denoted by $\operatorname{Owen}(N, D, \mathfrak{R})$. At times, if there is no confusion, we simply use $o$ to refer to the Owen point. Following Guardiola et al. (2008), it turns out that the Owen point is a core-allocation which can be reached through a population monotonic allocation scheme; hence every PI-game is a totally balanced game.

In some situations we will use $c^{(N, D, \Re)}(S)$ instead of $c(S)$, in order to denote that the game $(N, c)$ comes from the situation $(N, D, \mathfrak{R})$.

We denote by $\Upsilon$ the set of production-inventory situations $(N, D, \Re)$ defined in the universe of players $U$, being $n \geq 1, T \geq 1$ and $D$ an integer matrix. We say that a player $i \in N$ is essential if there exists $t \in\{1, \ldots, T\}$ with $d_{t}^{N \backslash\{i\}}>0$ such that $y_{t}^{*}(N \backslash\{i\})>y_{t}^{*}(N)$. The reader may note that an essential player is the one for which there exists at least one period in which he is needed by the rest of players in order to produce at a minimum cost a certain demand. We denote by $\mathcal{E}$ the set of essential players. The players not being essential are called inessential. It can be checked that for each inessential player $i, o_{N \backslash\{i\}}=c(N \backslash\{i\})$ holds. Finally, Guardiola et al. (2008) showed that the core of PI-games shrinks to the Owen point just only when all players are inessential for the PI-situation.

\section{PI-games and PMAS-games}

This section introduces a new class of TU-games closely related to PI-games, namely PMASgames. This class consists of all non-negative cost games allowing for pmasses. It turns out that the classes of PI-and PMAS-games coincide.

We start this section proving that the class of PI-games is closed under finite sums. This result will be used later in the proof of the main theorem of this section.

Proposition 3.1. The sum of PI-games defined on the same set of players $N$ is a PI-game.

Proof. Consider two PI-games $(N, \bar{c})$ and $(N, \hat{c})$ that arise from two PI-situations $(N, \bar{D}, \overline{\mathfrak{R}})$ and $(N, \hat{D}, \hat{\mathfrak{R}})$, respectively. Denote by $\bar{T}, \hat{T}$ the number of periods for the first and second PI-situations, respectively. Now, we build a new PI-situation $(N, D, \Re)$ with, 


$$
\begin{array}{r}
D=(\bar{D}|0| \hat{D})=\left(\begin{array}{ccccccccc}
\bar{d}_{1}^{1} & \bar{d}_{2}^{1} & \ldots & \bar{d}_{\bar{T}}^{1} & 0 & \hat{d}_{1}^{1} & \hat{d}_{2}^{1} & \ldots & \hat{d}_{\hat{T}}^{1} \\
\bar{d}_{1}^{2} & \bar{d}_{2}^{2} & \ldots & \bar{d}_{\bar{T}}^{2} & 0 & \hat{d}_{1}^{2} & \hat{d}_{2}^{2} & \ldots & \ldots \\
\vdots & \vdots & \vdots & \vdots & 0 & \vdots & \vdots & \vdots & \vdots \\
\bar{d}_{1}^{n} & \bar{d}_{2}^{n} & \ldots & \bar{d}_{\bar{T}}^{n} & 0 & \hat{d}_{1}^{n} & \hat{d}_{2}^{n} & \ldots & \hat{d}_{\hat{T}}^{n}
\end{array}\right) \\
H=(\bar{H}|\omega| \hat{H})=\left(\begin{array}{ccccccccc}
\bar{h}_{1}^{1} & \bar{h}_{2}^{1} & \ldots & \bar{h}_{\bar{T}}^{1} & \omega & \hat{h}_{1}^{1} & \hat{h}_{2}^{1} & \ldots & \hat{h}_{\hat{T}}^{1} \\
\bar{h}_{1}^{2} & \bar{h}_{2}^{2} & \ldots & \bar{h}_{\bar{T}}^{2} & \omega & \hat{h}_{1}^{2} & \hat{h}_{2}^{2} & \ldots & \hat{h}_{\hat{T}}^{2} \\
\vdots & \vdots & \vdots & \vdots & \omega & \vdots & \vdots & \vdots & \vdots \\
\bar{h}_{1}^{n} & \bar{h}_{2}^{n} & \ldots & \bar{h}_{\bar{T}}^{n} & \omega & \hat{h}_{1}^{n} & \hat{h}_{2}^{n} & \ldots & \hat{h}_{\hat{T}}^{n}
\end{array}\right)
\end{array}
$$

where $\omega \in \mathbb{R}$ is sufficiently large. Matrices $B$ and $P$ are defined in the same way as $H$. The PI-situation $(N, D, \Re)$ has $T=\bar{T}+\hat{T}+1$ periods. Take a coalition $S \subseteq N$ and consider $\bar{y}(S) \in R^{\bar{T}}, \hat{y}(S) \in R^{\hat{T}}$ being optimal solutions for the PI-situations $(N, \bar{D}, \overline{\mathfrak{R}})$ and $(N, \hat{D}, \hat{\mathfrak{R}})$, respectively. The reader may note that is not optimal satisfying the demand $d_{t}^{S}$ with $t \leq \bar{T}$ in a period $\tilde{t} \geq \bar{T}+1$ since backlogging costs are higher. By a similar argument the demand $d_{t}^{S}$ with $t>\bar{T}+1$ should not be satisfied from a period $\tilde{t} \leq \bar{T}$. Hence, the optimal solution for any coalition $S \subseteq N$ in a period $t \in\{1, \ldots T\}$ is given by

$$
y_{t}^{*}(S)=\left\{\begin{array}{l}
\bar{y}_{t}(S), \quad \text { if } t \leq \bar{T} \\
\omega, \quad \text { if } t=\bar{T}+1 \\
\hat{y}_{t}(S), \quad \text { if } \bar{T}+1<t \leq \hat{T}
\end{array}\right.
$$

being $\bar{y}_{t}(S)$ and $\hat{y}_{t}(S)$ as defined above. Hence, for each $S \subseteq N$,

$$
\bar{c}(S)+\hat{c}(S)=\sum_{t=1}^{\bar{T}} \bar{d}_{t}^{S} \bar{y}_{t}(S)+\sum_{t=1}^{\hat{T}} \hat{d}_{t}^{S} \hat{y}(S)=\sum_{t=1}^{T} d_{t}^{S} y_{t}^{*}(S)=c(S) .
$$

Next we define the concept of PMAS-game. Every non-negative cost game $(N, c)$ that possesses a PMAS is called a PMAS-game. The reader may note that the class of PMAS-games is a cone.

Our main result in this section states the relationship between PI-games and PMAS-games.

Theorem 3.2. Every PMAS-game is a PI-game.

Proof. Take $(N, c)$ a PMAS-game. Due to Proposition 3.1 we can assume that $c$ is an extreme direction of the cone of PMAS-games. Sprumont (1990) shows that (up to normalization) the set of extreme directions consists of all 0 -monotone simple veto games. Let $i_{v}$ be a veto player of $c$. Define $M=\left\{S \subset N \mid c(S)=1\right.$ and $c\left(S^{\prime}\right)=0$ for all $\left.S^{\prime} \supset S\right\}$, i.e., $M$ consists of all maximal coalitions with value 1 . Let $T=|M|+1$ and enumerate the elements of $M$, i.e., $M=\left\{S_{1}, \ldots, S_{T-1}\right\}$. Define $(N, D, \mathfrak{R})$ by

$$
\begin{aligned}
& d_{t}^{i}=\left\{\begin{array}{ll}
1 & \text { if }(i, t)=\left(i_{v}, T\right), \\
0 & \text { otherwise, }
\end{array} \quad b_{t}^{i}=1 \text { for all } i \text { and } t,\right. \\
& h_{t}^{i}=\left\{\begin{array}{ll}
1 & \text { if } t<T \text { and } i \in S_{t}, \\
0 & \text { otherwise }
\end{array}, \quad p_{t}^{i}= \begin{cases}0 & \text { if } t=1, \\
1 & \text { otherwise }\end{cases} \right.
\end{aligned}
$$


Let $(N, \bar{c})$ be the game generated by this PI-situation. We will show that $\bar{c}$ and $c$ coincide. Let $S \subseteq N$. If $i_{v} \notin S$, then $\bar{c}(S)=c(S)=0$, since $\sum_{j \in S} d^{j}=0$ and $i_{v}$ is a veto player of $c$. If $i_{v} \in S$, then $S$ has to produce one unit. If period $T$ is chosen to do this, it will cost 1 . Hence, $\bar{c}(S) \in\{0,1\}$. Coalition $S$ can (only) produce freely in the first period. It can fulfill the demand for free if it has no holding costs in each period (but the last). This is the case if and only if $S \nsubseteq S_{t}$ for every $t \in\{1, \ldots, T-1\}$. On the other hand, because $c$ is 0 -monotone, $c(S)=1$ if and only if $S$ is a subset of some maximal coalition with value 1 . We conclude that $\bar{c}$ and $c$ coincide.

We conclude this section with a nice relationship between PI-games and concave games.

Corollary 3.3. Each non-negative concave game is a PI-game.

Proof. It is follows directly from the fact that any non-negative concave game has a PMAS: the Shapley value.

\section{Characterizations of the Owen point}

This section is devoted to address the second goal of this paper, namely to provide axiomatic foundations for the Owen point. Axiomatic characterizations of solution concepts in game theory is a fruitful area of research. The interested reader is referred to Peleg and Sudhöter (2003) and Moulin (1988) for a comprehensive study of this field.

A solution rule $\varphi$ on $\Upsilon$ is a map, which assigns to every production-inventory situation $(N, D, \mathfrak{R}) \in \Upsilon$ a subset of $\mathbb{R}^{N}$. In particular, the core and the Owen set are solution rules. (Recall that the core was introduced by Gilles (1959) and the Owen set by Owen (1975).)

Gellekom et al. (2000) prove that the Owen set as considered as a solution rule is not "gametheoretical". Thus, first of all we wonder whether the Owen point exhibits the same behavior.

Our next example shows that the Owen point is not a game-theoretical solution since it depends on PI-situations and not on PI-games.

Example 4.1. Consider the following PI-situation $(N, D, \Re) \in \Upsilon$ with two periods and two players, namely $P 1$ and $P 2$ :

\begin{tabular}{ccccccc}
\hline & \multicolumn{2}{c}{ Demand } & \multicolumn{2}{c}{ Production } & Inventory & Backlogging \\
\hline P1 & 1 & 0 & 2 & 1 & 1 & 1 \\
P2 & 0 & 2 & 1 & 1 & 2 & 2 \\
\hline
\end{tabular}

The data above gives rise to the game with characteristic function in the following table:

\begin{tabular}{llllllll}
\hline & $d_{1}^{S}$ & $d_{2}^{S}$ & $p_{1}^{S}$ & $p_{2}^{S}$ & $h_{1}^{S}$ & $b_{1}^{S}$ & $c$ \\
\hline 1$\}$ & 1 & 0 & 2 & 1 & 1 & 1 & 2 \\
$\{2\}$ & 0 & 2 & 1 & 1 & 2 & 2 & 2 \\
$\{1,2\}$ & 1 & 2 & 1 & 1 & 1 & 1 & 3 \\
\hline
\end{tabular}

This gives $\operatorname{Owen}(N, D, \mathfrak{R})=\{(1,2)\}$. On the other hand, the PI-situation $\left(N, D^{\prime}, \Re^{\prime}\right) \in \Upsilon$, where 


\begin{tabular}{ccccccc}
\hline & \multicolumn{2}{c}{ Demand } & \multicolumn{2}{c}{ Production } & Inventory & Backlogging \\
\hline P1 & 0 & 2 & 1 & 1 & 2 & 2 \\
P2 & 1 & 0 & 2 & 1 & 3 & 2 \\
\hline
\end{tabular}

provides the same PI-game, but now the Owen point is different Owen $\left(N, D^{\prime}, \mathfrak{R}^{\prime}\right)=\{(2,1)\}$.

In spite of the behavior shown by Example 4.1, the Owen point is an attractive cost sharing vector in PI-games. Actually, it is the unique core-allocation reached by dual solutions within the class of PI-games. Moreover, there is always a PMAS that realizes the Owen point. Therefore, our goal in the rest of the section is to find different axiomatic characterizations for the Owen point.

Let $\varphi$ be a solution rule on $\Upsilon$, we consider the following properties:

(EF) Efficiency. For all $y \in \varphi(N, D, \Re)$ and for all $(N, D, \Re) \in \Upsilon, y_{N}=c^{(N, D, \Re)}(N)$.

(NE) Non-emptiness. For all $(N, D, \mathfrak{R}) \in \Upsilon, \varphi(N, D, \mathfrak{R}) \neq \varnothing$.

(PO) Positivity. For all $(N, D, \mathfrak{R}) \in \Upsilon$ and for all $y \in \varphi(N, D, \Re), y_{i} \geq 0$ for each $i \in N$.

(IR) Individual rationality. For all $(N, D, \mathfrak{R}) \in \Upsilon$, for all $y \in \varphi(N, D, \mathfrak{R})$, and for all $i \in N, y_{i} \leq c^{(N, D, \Re)}(\{i\})$.

(IE) Inessentiality. For all $(N, D, \mathfrak{R}) \in \Upsilon$ and for all $y \in \varphi(N, D, \mathfrak{R})$, if $i$ is an inessential player for $(N, D, \Re)$, then $y_{N \backslash\{i\}} \leq c^{(N, D, \Re)}(N \backslash\{i\})$.

(AP) Additivity of players' demands. For all $(N, D, \mathfrak{R}) \in \Upsilon$ and for all $y \in \varphi(N, D, \mathfrak{R})$, there exists $\left(z_{k}\right)_{k \in N} \in\left(\mathbb{R}^{N}\right)^{N}$ such that $y=\sum_{k \in N} z_{k}$ and for all $k \in N, z_{k} \in \varphi\left(N, D_{k}, \mathfrak{R}\right)$, where

$$
D_{k}=\left(d^{i t}\right)_{\substack{i=1, \ldots, n, t=1, \ldots, T}}, \quad d^{i t}= \begin{cases}d_{t}^{i} & \text { if } k=i, \\ 0 & \text { otherwise }\end{cases}
$$

Efficiency guarantees that the overall cost of any PI-situation is entirely divided among the players. Non-emptiness assures that the solution rule will never return the empty set as an admissible allocation. Positivity ensures that the allocation process of the overall saving does not compensate those players that have zero demand for all periods (subsidizing players is not allowed). On the contrary, it forces the remaining players to pay their own demands. Individual rationality protects all players from supporting a higher cost than what they generate by themselves. Inessentiality imposes collective rationality for every coalition in which an inessential player has left.

Finally, a solution rule satisfies additivity of players' demands property if it is additive for the demand of each individual player; i.e. it is additive with respect to matrices where just a player keeps its demand and the remaining demands are set to zero. Note that this particular form of additivity arises from the fact that $c^{(N, D, \Re)}=\sum_{i \in N} c^{\left(N, D_{i}, \mathfrak{R}\right)}$ for all $(N, D, \Re) \in \Upsilon$. Therefore, we focus on those solution rules for PI-situations that are consistent with this kind of partition of demands.

In order to get some insights into the above properties, the reader can easily check that the Shapley value satisfies EF, NE and AP. In the following, we also prove that the Owen point satisfies all the above properties.

Proposition 4.2. On $\Upsilon$, the Owen point satisfies EF, NE, PO, IR, IE, and AP. 
Proof. For all $(N, D, \mathfrak{R}) \in \Upsilon$ we have that $\operatorname{Owen}(N, D, \mathfrak{R}) \in \operatorname{Core}(N, c)$. Then the Owen point satisfies EF, IR, IE and NE. Moreover, since all costs and demands are non-negatives, $\operatorname{Owen}_{i}(N, D, \Re)=\sum_{t=1}^{T} d_{t}^{i} y_{t}^{*}(N) \geq 0$ for each $i \in N$. Therefore, the Owen point satisfies PO.

Finally, if we define $D_{i}$ as in (1), we have that $\sum_{i \in N} D_{i}=D$ and

$$
\begin{aligned}
\operatorname{Owen}(N, D, \mathfrak{R}) & =D y^{*}(N)=\left(\sum_{i \in N} D_{i}\right) y^{*}(N) \\
& =\sum_{i \in N}\left(D_{i} y^{*}(N)\right)=\sum_{i \in N} \operatorname{Owen}\left(N, D_{i}, \mathfrak{R}\right) .
\end{aligned}
$$

Hence, the Owen point satisfies AP.

Now, we can prove that if all players are inessential, the Owen point can be characterized just by three of the above properties.

Proposition 4.3. Let $(N, D, \Re) \in \Upsilon$ such that $|\mathcal{E}|=0$. The solution rule $\varphi$ on $\Upsilon$ satisfies $E F$, $N E$, and $I E$ if and only if $\varphi(N, D, \mathfrak{R})=\operatorname{Owen}(N, D, \mathfrak{R})$.

Proof. (If) Immediately follows by Proposition 4.2.

(Only if) By NE, $\varphi(N, D, \mathfrak{R}) \neq \varnothing$ and by EF, $y_{N}=c^{(N, D, \mathfrak{R})}(N)$ for all $y \in \varphi(N, D, \mathfrak{R})$.

Take $y \in \varphi(N, D, \mathfrak{R})$. Since all players $i \in N$ are inessential, by IE, it holds that $y_{N \backslash\{i\}} \leq$ $c^{(N, D, \mathfrak{R})}(N \backslash\{i\})=o_{N \backslash\{i\}}$ for each $i \in N$. Hence, $y_{i} \geq c^{(N, D, \mathfrak{R})}(N)-c^{(N, D, \Re)}(N \backslash\{i\})=$ $o_{N}-o_{N \backslash\{i\}}=o_{i}$ for all $i \in N$. Therefore by $\operatorname{EF,} \varphi(N, D, \mathfrak{R})=\operatorname{Owen}(N, D, \mathfrak{R})$.

The next two results of this section state that, in general, characterizing the Owen point for a number of players $n$, (arbitrary but fixed) can be done with different combinations of the above six properties.

Theorem 4.4. Let $(N, D, \mathfrak{R}) \in \Upsilon$. An allocation rule on $(N, D, \mathfrak{R})$ satisfies $E F, N E, P O, I E$, and $A P$ if and only if it coincides with the Owen point.

Proof. (If) Follows from Proposition 4.2.

(Only if) Let $\varphi$ be an allocation rule. If $|\mathcal{E}|=0$ by Proposition $4.3 \varphi(N, D, \mathfrak{R})=$ $\operatorname{Owen}(N, D, \mathfrak{R})$. Therefore, we suppose that $|\mathcal{E}| \geq 1$. We have that $D=D_{1}+D_{2}+\cdots+D_{n}$ where $D_{i}$ is (see (1)):

$$
D_{i}=\left(\begin{array}{cccc}
0 & 0 & \cdots & 0 \\
\vdots & \vdots & \vdots & \vdots \\
0 & 0 & \cdots & 0 \\
d_{1}^{i} & d_{2}^{i} & \cdots & d_{T}^{i} \\
0 & 0 & \cdots & 0 \\
\vdots & \vdots & \vdots & \vdots \\
0 & 0 & \cdots & 0
\end{array}\right) .
$$

Then for all $i \in N,\left(N, D_{i}, \mathfrak{R}\right)$ is a PI-situation with $D_{i}$ an integer matrix and therefore it belongs to $\Upsilon$. Take $i \in N$, then the Owen point for $\left(N, D_{i}, \mathfrak{R}\right)$ is given by $\left(o_{k}\right)_{k=1, \ldots, n}$ :

$$
o_{k}= \begin{cases}\sum_{t=1}^{T} d_{t}^{i} y_{t}^{*}(N) & \text { if } k=i, \\ 0 & \text { otherwise. }\end{cases}
$$


By NE, $\varphi\left(N, D_{i}, \mathfrak{R}\right) \neq \varnothing$. Take $y \in \varphi\left(N, D_{i}, \mathfrak{R}\right)$, the player $i$ is inessential for this situation, since there is no $t \in\{1, \ldots, T\}$ with $d_{t}^{N \backslash\{i\}}>0$ such that $y_{t}^{*}(N \backslash\{i\})<y_{t}^{*}(N)$. Moreover, $c^{\left(N, D_{i}, \mathfrak{R}\right)}(N \backslash\{i\})=0$. By IE $y_{N \backslash\{i\}} \leq c^{\left(N, D_{i}, \mathfrak{R}\right)}(N \backslash\{i\})=0$. Therefore, by PO, $y_{j}=0$ for each $j \in N \backslash\{i\}$. Finally, by EF, $y=o$ and hence $\varphi\left(N, D_{i}, \mathfrak{R}\right)=\operatorname{Owen}\left(N, D_{i}, \mathfrak{R}\right)$. Thus, if $y \in \varphi(N, D, \Re)$ by AP it follows that $y=z_{1}+\cdots+z_{n}$ with $z_{i} \in \varphi\left(N, D_{i}, \mathfrak{R}\right)$ for all $i \in N$, and so

$$
y=\sum_{i \in N} z_{i}=\sum_{i \in N} \operatorname{Owen}\left(N, D_{i}, \mathfrak{R}\right)=\operatorname{Owen}(N, D, \mathfrak{R}) .
$$

Hence, we conclude that $\varphi(N, D, \mathfrak{R})=\operatorname{Owen}(N, D, \mathfrak{R})$.

Remark 4.5. The reader may note that Theorem 4.4 could be proved without using Proposition 4.3. However, we propose this alternative proof in order to stress that for those PI-situations where all players are inessential, the Owen point (and hence the core) can be characterized by just three properties.

Remark 4.6. An alternative characterization for the Owen point can be obtained just swapping properties IE and IR. It is clear that the Owen point satisfies EF, NE, PO, IR, and AP. Now, let $\varphi$ be an allocation rule. By NE, an allocation $y \in \varphi\left(N, D_{i}, \mathfrak{R}\right)$ exists. Note that $c^{\left(N, D_{i}, \mathfrak{R}\right)}(\{j\})=0$ for all $j \in N \backslash\{i\}$ then by PO and IR, $y_{j}=0$ for each $j \in N \backslash\{i\}$. Finally, by EF, $y_{i}=c^{\left(N, D_{i}, \mathfrak{R}\right)}(N)=\sum_{t=1}^{T} d_{t}^{i} y_{t}^{*}(N)$ and hence $\varphi\left(N, D_{i}, \mathfrak{R}\right)=\operatorname{Owen}\left(N, D_{i}, \mathfrak{R}\right)$. Since both $\varphi$ and the Owen point satisfy AP it follows that $\varphi(N, D, \Re)=\operatorname{Owen}(N, D, \Re)$.

In the following, we prove that the two sets of axioms used in Theorem 4.4 and Remark 4.6 are logically independent.

Example 4.7. Consider $\varphi$ on $\Upsilon$ defined by

$$
\varphi(N, D, \mathfrak{R}):=\left\{\begin{array}{l}
\left\{\begin{array}{l}
\left(\frac{c^{(N, D, \Re)}(N)}{2}, \frac{c^{(N, D, \Re)}(N)}{2}\right), \\
\operatorname{Owen}(N, D, \mathfrak{R}) \\
\text { Owen }(N, D, \mathfrak{R}),
\end{array}\right. \\
\text { otherwise, }
\end{array}\right.
$$

where

$$
\Upsilon^{1}:=\left\{(N, D, \Re) \in \Upsilon / \quad \begin{array}{c}
|N|=T=2, \\
c^{(N, D, \mathfrak{R})}(N) \leq c^{(N, D, \mathfrak{R})}(\{i\}) \quad \forall i \in N
\end{array}\right\} .
$$

$\varphi(N, D, \Re)$ satisfies NE, EF, PO, IR and IE but not AP.

Example 4.8. We take $\varphi$ on $\Upsilon$ defined by

$$
\varphi(N, D, \mathfrak{R}):=\left\{\left(c^{(N, D, \Re)}(N), 0,0, \ldots, 0\right) \in \mathbb{R}^{N}\right\} .
$$

$\varphi(N, D, \Re)$ satisfies NE, EF, PO and AP but neither IR nor IE.

Example 4.9. Take $\varphi$ on $\Upsilon$ given by

$$
\varphi(N, D, \mathfrak{R}):= \begin{cases}\left(p_{1}^{1} d_{1}^{1}+\left(p_{1}^{1}-p_{1}^{2}\right) d_{1}^{2}, p_{1}^{2} d_{1}^{2}\right), & (N, D, \mathfrak{R}) \in \Upsilon^{2} \\ \text { Owen }(N, D, \mathfrak{R}), & \text { otherwise, }\end{cases}
$$


where

$$
\Upsilon^{2}:=\left\{(N, D, \Re) \in \Upsilon /|N|=2, T=1, p_{1}^{1}<p_{1}^{2}\right\} .
$$

$\varphi(N, D, \Re)$ satisfies NE, EF, IR, IE and AP but not PO.

Example 4.10. Let $\varphi$ on $\Upsilon$ be defined by

$$
\varphi(N, D, \mathfrak{R}):=\left\{(0,0, \ldots, 0) \in \mathbb{R}^{N}\right\}
$$

$\varphi(N, D, \Re)$ satisfies NE, PO, IR, IE and AP but not EF.

Example 4.11. Define $\varphi$ on $\Upsilon$ by

$$
\varphi(N, D, \mathfrak{R}):=\varnothing .
$$

$\varphi(N, D, \Re)$ satisfies EF, PO, IR, IE and AP but not NE.

To conclude this section we provide an alternative characterization for the Owen point, based on a different rationale: consistency. In the previous section we proved that the class of PI-games coincides with the class of non-negative cost games with pmasses. Here, we will use a consistency property on population monotonicity (see Thomson (1995) for further details on population monotonic solution rules) that allows us to characterize the Owen point. This characterization is similar to the one given in Grafe et al. (1998) when studying the proportional rule on the class of externality games (see Proposition 4.2 in Grafe et al. (1998)) and is based on the following properties.

(PM) Population monotonicity. A solution rule $\varphi$ defined on $\Upsilon$ is said to satisfy PM if for all $(N, D, \mathfrak{R}) \in \Upsilon$, for all $S \subseteq N$, for all $y \in \varphi(N, D, \mathfrak{R}), z \in \varphi\left(S, D_{S}, \mathfrak{R}_{S}\right)$, and for all $i \in S$, we have $y_{i} \leq z_{i}$, being $\left(S, D_{S}, \Re_{S}\right) \in \Upsilon$ derived from $(N, D, \Re)$ by restricting each matrix to the members of $S$.

(AN) Anonymity property. A solution rule $\varphi$ defined on $\Upsilon$ is said to satisfy AN if for every PIsituations $(N, D, \mathfrak{R})$, every bijection $\sigma: N \rightarrow N^{\prime}$, and every $y \in \varphi(N, D, \mathfrak{R})$, we have $z \in\left(N^{\prime}, D^{\prime}, \mathfrak{R}^{\prime}\right)$, where $y_{i}=z_{\sigma(i)}$ and $\sigma(i)$ has the demands and costs in $\left(N^{\prime}, D^{\prime}, \Re^{\prime}\right)$ that $i$ has in $(N, D, \Re)$, for all $i \in N$.

The last result of this section proves that there is a unique non-empty, efficient and anonymous solution rule on the set of production-inventory situations that satisfies population monotonicity: the Owen point.

Theorem 4.12. An allocation rule on $\Upsilon$ satisfies EF, NE, PM, and AN if and only if it is the Owen point.

Proof. (If) It is clear that the Owen point satisfies EF, NE, AN and PM.

(Only if) Let $\varphi$ be an allocation rule on $\Upsilon$ that satisfies EF, NE, PM, and AN. We assume that $\varphi \neq$ Owen. Then there exists a PI-situation $(N, D, \Re)$ such that for some $i \in N$ and for some $y \in \varphi(N, D, \Re), y_{i} \neq \operatorname{Owen}_{i}(N, D, \mathfrak{R})$. By EF, one has that $n \geq 2$. Besides, again by EF, there has to be a player $j \in N$ such that $y_{j}<\operatorname{Owen}_{j}(N, D, \Re)$.

Let $k$ be a player not in $N$. Consider a PI-situation $(\tilde{N}, \tilde{D}, \tilde{\Re})$ where $\tilde{N}=N \cup\{k\}$ and for each $i \in \tilde{N} \backslash\{k\}, \tilde{D}_{i}=D_{i}, \tilde{\Re}_{i}=\Re_{i}$ and $\tilde{D}_{k}=D_{j}, \tilde{\Re}_{k}=\Re_{j}$. Then $y_{t}^{*}(N)=\tilde{y}_{t}^{*}(\tilde{N})$ for all $t \in T$. Take $z \in \varphi(\tilde{N}, \tilde{D}, \tilde{\mathfrak{R}})$. In addition, by PM, $z_{i} \leq y_{i}$, for each $i \in N$. Moreover, by AN, 
$z_{j}=z_{k}$, so $z_{k}<\operatorname{Owen}_{j}(N, D, \Re)$. Then by EF we obtain:

$$
\sum_{t=1}^{T} \tilde{d}_{t}^{\tilde{N}} y_{t}^{*}(N)=\sum_{t=1}^{T} \tilde{d}_{t}^{\tilde{N}} \tilde{y}^{*}(\tilde{N})=\sum_{i \in \tilde{N}} z_{i} .
$$

Furthermore by EF and PM we obtain:

$$
\begin{aligned}
\sum_{i \in \tilde{N}} z_{i} & =z_{k}+z_{j}+\sum_{i \in N \backslash\{j\}} z_{i} \leq y_{j}+y_{j}+\sum_{i \in N \backslash\{j\}} y_{i} \\
& <\operatorname{Owen}_{j}(N, D, \Re)+\sum_{i \in N} \operatorname{Owen}_{i}(N, D, \Re)=\sum_{t=1}^{T} \tilde{d}_{t}^{\tilde{N}} y_{t}^{*}(N) .
\end{aligned}
$$

Consequently there is a contradiction.

\section{Concluding remarks}

In this paper we have revisited the class of production-inventory games, focussing on two of the open problems proposed in Guardiola et al. (2008): (1) Studying relationships of the class of PI-games with other classes of games and (2) Finding axiomatic characterizations of the Owen point. First of all, we have introduced the class of non-negative games with pmasses to prove that PI-games and PMAS-games coincide. Regarding the second point, we have introduced a set of axioms: efficiency, non-emptiness, positivity, inessentiality, individual rationality, additivity of players' demands, anonymity, and population monotonicity that provide three different characterizations of the Owen point for production-inventory situations.

\section{References}

Anupindi, R., Bassok, Y., Zemel, E., 2001. A general framework for the study of decentralized distribution systems. Manuf. Service Oper. Manag. 3, 349-368.

Bondareva, O.N., 1963. Some applications of linear programming methods to the theory of cooperative games. Problemy Kibernety 10, 119-139.

Borm, P.E.M., Hamers, H., Hendrickx, R., 2001. Operations research games: A survey. TOP 9, 139-216.

Deng, X., Ibaraki, T., Nagamochi, H., 1999. Algorithmic aspect of the core of combinatorial optimization games. Math. Oper. Res. 24, 751-766.

Deng, X., Ibaraki, T., Nagamochi, H., Zang, W., 2000. Totally balanced combinatorial optimization games. Math. Program. 87, 441-452.

Eppen, G.D., 1979. Effect of centralization on expected cost in a multi-location newsboy problem. Manag. Sci. 25, 498-501.

Gellekom, J.R.G., Potters, J.A.M., Reijnierse, J.H., Engel, M.C., Tijs, S.H., 2000. Characterization of the Owen set of linear production processes. Games Econom. Behav. 32, 139-156.

Gerchak, Y., Gupta, D., 1991. On apportioning costs to customers in centralized continuous review inventory systems. J. Oper. Manag. 10, 546-551.

Gilles, D.B., 1959. Solutions to general non-zero-sum games. In: Contributions to the Theory of Games vol. IV. In: Annals of Math. Studies, vol. 40. pp. 47-85.

Grafe, F., Iñarra, E., Zarzuelo, J.M., 1998. Population monotonic allocation schemes on externality games. Math. Methods Oper. Res. 48, 71-80.

Guardiola, L.A., Meca, A., Puerto, J., 2008. Production-inventory games: A new class of totally balanced combinatorial optimization games. Games Econom. Behav. doi:10.1016/j.geb.2007.02.003.

Hartman, B.C., Dror, M., 2003. Optimizing centralized inventory operations in a cooperative game theory setting. IIE Trans. Oper. Eng. 35, 243-257.

Hartman, B.C., Dror, M., 2005. Allocation of gains from inventory centralization in newsvendor environments. IIE Trans. Scheduling Logist. 37, 93-107. 
Hartman, B.C., Dror, M., 1996. Cost allocation in continuous review inventory models. Naval Res. Logist. 43, 549-561.

Hartman, B.C., Dror, M., Shaked, M., 2000. Cores of inventory centralization games. Games Econom. Behav. 31, $26-49$.

Meca, A., 2007. A core-allocation family for generalized holding cost games. Math. Methods Oper. Res. 65, $499-517$.

Meca, A., Timmer, J, García-Jurado, I., Borm, P.E.M., 2004. Inventory games. European J. Oper. Res. 156, $127-139$.

Meca, A., García-Jurado, I., Borm, P.E.M., 2003. Cooperation and competition in inventory games. Math. Methods Oper. Res. 57, 481-493.

Meca, A., Guardiola, L.A., Toledo, A., 2007. p-additive games: A class of totally balanced games arising from inventory situations with temporary discounts. TOP $15,322-340$.

Minner, S., 2006. Bargaining for cooperative economic ordering. Decis. Support Syst. 43, 569-583.

Moulin, H., 1988. Axioms of Cooperative Decision Making. Cambridge U. Press, New York.

Müller, A., Scarsini, M., Shaked, M., 2002. The newsvendor game has a nonempty core. Games Econom. Behav. 38, 118-126.

Owen, G., 1975. On the core of linear production games. Math. Program. 9, 358-370.

Peleg, B., Sudhöter, P., 2003. Introduction to the Theory of Cooperative Games. Kluwer Academic, Boston.

Robinson, L.W., 1993. Comment on "On apportioning costs to customers in centralized continuous review inventory systems”, by Gerchak and Gupta. J. Oper. Manag. 11, 99-102.

Shapley, L.S., 1967. On balanced sets and cores. Naval Res. Logist. 14, 453-460.

Shapley, L.S., 1971. Cores of convex games. Int. J. Game Theory 1, 11-26.

Shapley, L.S., Shubik, M., 1969. On market games. J. Econom. Theory 1, 9-25.

Slikker, M., Fransoo, J., Wouters, M., 2005. Cooperation between multiple news-vendors with transshipments. European J. Oper. Res. 167, 370-380.

Sprumont, Y., 1990. Population monotonic allocation schemes for cooperative games with transferable utility. Games Econom. Behav. 2, 378-394.

Thomson, W., 1995. Population-monotonic allocation rules. In: Barnett, W., Moulin, H., Salles, M., Chofield, N. (Eds.), Social Choice, Welfare and Ethics. Cambridge University Press, pp. 79-124 (Chapter 4).

Tijs, S.H., Meca, A., López, M.A., 2005. Benefit sharing in holding situations. European J. Oper. Res. 162, $251-269$.

Wagner, H.M., Whitin, T.M., 1958. Dynamic version of the economic lot size model. Manag. Sci. 5, 89-96. 